\title{
FENOLOGIA DE Astronium graveolens Jacq. EM FLORESTA ESTACIONAL DECIDUAL EM VITÓRIA DA CONQUISTA, BAHIA
}

\author{
PHENOLOGY OF Astronium graveolens Jacq. IN SEASONAL DECIDUOUS FOREST IN VITÓRIA \\ DA CONQUISTA, BAHIA
}

\author{
Rodrigo Lacerda Brito Neto ${ }^{1}$ Emerson Iuri de Paula Araújo ${ }^{1}$ Cássio Maggi Salvia Maciel ${ }^{2}$ \\ Alessandro de Paula ${ }^{3}$ Cristiano Tagliaferre ${ }^{4}$
}

\begin{abstract}
RESUMO
O presente trabalho teve como objetivo estudar a fenologia da espécie arbórea Astronium graveolens Jacq. e correlacioná-la com a precipitação, umidade relativa e temperatura da região de estudo. Os estudos foram conduzidos em um fragmento de floresta estacional decidual, no campus da Universidade Estadual do Sudoeste da Bahia em Vitória da Conquista, Bahia, Brasil. Os indivíduos foram visitados quinzenalmente de setembro de 2010 a dezembro de 2014, quando foram registradas as características fenológicas referentes a desfolhamento, brotamento, floração e frutificação. A análise dos dados fenológicos foi feita através de testes de normalidade, utilização de estatística circular para testar a sazonalidade das fenofases e teste de correlação de postos de Spearman, para verificar com qual intensidade e sentido os eventos climáticos se relacionavam com as fenofases. A espécie apresentou uma queda foliar com padrão decíduo de alta sincronia com concentração no período de seca, seguido pelo brotamento altamente sincrônico que se iniciou no começo da estação úmida. A floração e frutificação apresentaram um padrão bianual, sendo um evento que se manifestou como sendo de baixa sincronia a assincrônico.
\end{abstract}

Palavras-chave: sincronia; fenofase; clima.

\begin{abstract}
This current study had the objective to study the phenology of tree species Astronium graveolens Jacq. and correlates it with precipitation, relative humidity and temperature of the study area. The studies were conducted in a fragment of the deciduous forest in the campus of the State University of Southwest Bahia in Vitória da Conquista, Bahia state, Brazil. The subjects were visited fortnightly from September 2010 to December 2014, when the phenological characteristics related to senescence, budding, flowering and fruiting were recorded. Data analysis done through normality tests, utilization of circular statistics to test the seasonality of phenophases and correlation test of Spearman's rank to check with which intensity and direction the climatic events were related to the phenophases. The species presented a foliar fall with deciduous pattern of high synchrony with concentration in the dry season, followed by budding highly synchronic that began in the early wet season. The flowering and fruiting presented a biennial pattern, being an event that was manifested as low sync at the asynchronous.
\end{abstract}

Keywords: sync; phenophase; weather.

1 Engenheiro Florestal, Mestrando do Programa de Pós-Graduação em Ciências Florestais, Universidade Estadual do Sudoeste da Bahia, Estrada do Bem Querer, Km 4, CEP 45083-900, Vitória da Conquista (BA), Brasil. lacerdadm@hotmail.com / eng.iuri90@gmail.com

2 Engenheiro Florestal, MSc., Gestor Ambiental na Secretaria de Estado de Meio Ambiente e Desenvolvimento Sustentável de Minas Gerais, Rua Agapito dos Anjos, 455, CEP 39401-040, Montes Claros (MG), Brasil. macielflorestal@hotmail.com

3 Engenheiro Florestal, Dr., Professor do Programa de Pós-Graduação em Ciências Florestais, Universidade Estadual do Sudoeste da Bahia, Estrada do Bem Querer, Km 4, CEP 45083-900, Vitória da Conquista (BA), Brasil. apaula@uesb.edu.br

4 Engenheiro Agrônomo, Dr., Professor do Programa de Pós-Graduação em Ciências Florestais, Universidade Estadual do Sudoeste da Bahia, Estrada do Bem Querer, Km 4, CEP 45083-900, Vitória da Conquista (BA), Brasil. tagliaferre@yahoo.com.br

Recebido para publicação em 17/09/2015 e aceito em 7/04/2017

Ci. Fl., v. 28, n. 2, abr .- jun., 2018 


\section{INTRODUÇÃO}

Fenologia é o estudo das fases ou atividades do ciclo de vida de plantas ou animais e sua ocorrência temporal ao longo do ano, contribuindo para o entendimento dos padrões reprodutivos e vegetativos (MORELLATO, 1995), e das causas de sua ocorrência em relação às forças seletivas bióticas e abióticas. É empregada de maneira complementar às análises de anatomia vegetal (LISI et al., 2008), serve de base para a construção de planos de manejo, bem como para a elaboração de um projeto que vise à manutenção de espécies ameaçadas de extinção (SILVA; SANTOS, 2007) e ainda para avaliação da vulnerabilidade de espécies vegetais a incêndios florestais (LUCENA; LEITE; SILVA MATOS, 2015). Estudos fenológicos beneficiam a compreensão da dinâmica florestal, reunindo informações sobre o estabelecimento de espécies, como: o período de crescimento, de reprodução e a disponibilidade de recursos alimentares. Demonstram como as espécies reagem à atuação dos fatores climáticos, sendo que estes juntamente com as características intrínsecas das plantas, exercem influência sobre a periodicidade pela qual os eventos fenológicos se manifestam (FOURNIER, 1974; MORELLATO et al., 1990; BORCHERT et al., 2005).

A Astronium graveolens é uma espécie da família Anacardiaceae, sua altura está entre 15 e 25 $\mathrm{m}$, apresenta tronco liso, com diâmetro de 40 a $60 \mathrm{~cm}$, é uma espécie dióica com sistema de cruzamento obrigatoriamente alógamo em que a polinização é entomófila (ALLEM, 1991; LORENZI, 2008). Tem um bom crescimento em florestas primárias e secundárias sendo pouco frequente em áreas abertas, nas quais atinge menores dimensões. Trata-se de uma espécie plástica que ocupa diversos tipos de ambientes, sua distribuição natural vai desde o México até o Brasil, Bolívia e Paraguai (BARRANCE et al., 2003). Em relação à importância comercial, Hernandez et al. (2013) demonstraram um potencial de Astronium graveolens para a indústria farmacêutica, ao comprovar a capacidade antibacteriana do seu óleo essencial.

Estudos sobre a fenologia de Astronium graveolens já foram realizados por Reich e Borchert (1984) em uma floresta estacional decidual no interior da Costa Rica e por Paiva (2014) em uma floresta estacional semidecidual no sul do Espírito Santo, ambos com um ano de coleta de dados, e ainda por Morellato (1991) em uma floresta estacional semidecidual no interior do estado de São Paulo no Brasil com três anos de coleta de dados. São escassos os artigos científicos sobre a fenologia de Astronium graveolens para a região nordeste do Brasil, ou que contenham um período de coleta de dados superior a três anos.

Diante de seu potencial de uso, tanto ecológico quanto comercial, a espécie Astronium graveolens teve sua fenologia avaliada com os objetivos de: identificar a intensidade, sincronia, os padrões e os períodos de ocorrência de cada fenofase; e ainda a intensidade de correlação destas com as variáveis climáticas, fornecendo dados para auxiliar em planos de manejo que venham a utilizar a espécie, bem como para complementar os estudos fenológicos em florestas estacionais decíduas na região nordeste.

\section{MATERIAL E MÉTODO}

\section{Caracterização da área de estudo}

A área de estudo situa-se em Vitória da Conquista - BA, a $891 \mathrm{~m}$ de altitude, nas coordenadas geográficas: $-14^{\circ} 52^{\prime} 46^{\prime \prime}$ de latitude Sul e $-40^{\circ} 47^{\prime} 34$ " de longitude Oeste. Trata-se de um fragmento de floresta estacional decidual, regionalmente conhecida como mata de cipó, no campus da Universidade Estadual do Sudoeste da Bahia.

A mata de cipó do planalto da conquista é uma floresta relativamente baixa, por localizar-se em um planalto, possui relevo de baixa inclinação. Possui algumas clareiras e as árvores com alturas que variam entre 10 e 15 metros, apresentando um gradiente de caducifólia que vai de decidual (50\%) à semidecidual (20\%), (SOARES FILHO, 2000).

Segundo Jesus (2010), Vitória da Conquista caracteriza-se por possuir um clima semiárido e em algumas regiões subúmido, possuindo temperatura média anual de $19,6^{\circ} \mathrm{C}$. O período de maior precipitação de chuvas ocorre entre os meses de novembro e janeiro. O autor relatou ainda que esta região tem risco de seca que varia de alto a médio, possuindo $100 \%$ de sua área inserida no polígono das secas. 


\section{Obtenção dos dados}

Os dados climáticos foram obtidos na Estação Meteorológica de Vitória da Conquista, localizada nas coordenadas - $14^{\circ} 53^{\prime}$ de latitude Sul e - $40^{\circ} 8^{\prime}$ de longitude Oeste, a 874 m s.n.m. Foram calculadas as médias mensais dos valores diários para temperatura diária média e umidade relativa do ar, bem como a precipitação total mensal entre maio de 2010 a dezembro de 2014.

Para observar os eventos fenológicos utilizou-se uma trilha de aproximadamente um metro de largura já existente na área de estudo, os indivíduos foram selecionados no interior do fragmento e a $10 \mathrm{~m}$ de distância da trilha, evitando-se ainda as clareiras. Selecionaram-se 10 indivíduos que foram marcados com plaquetas de metal inoxidável numeradas segundo a ordem de aparecimento na trilha (FOURNIER, 1976), que apresentassem diâmetro a altura do peito (DAP) maior que $10 \mathrm{~cm}$. Suas fenofases foram avaliadas quinzenalmente com o auxílio de um binóculo e de uma ficha de campo para anotação dos dados. As observações foram feitas no período de $1^{\circ}$ de setembro de 2010 a 31 de dezembro de 2014.

Os parâmetros fenológicos observados foram: queda foliar (período em que as folhas mudam de cor e caem com facilidade); brotação (período que se inicia com o surgimento de pequenas folhas nos ápices dos ramos, e termina quando as folhas atingem o tamanho e coloração característicos); flores (período em que a árvore apresenta flores em antese ou botão); fruto (inicia quando é possível visualizar os frutículos após a fertilização das flores e termina com a dispersão das sementes).

\section{Análise dos dados}

Para classificar os meses em período seco e úmido, os dados mensais de precipitação total, umidade relativa média e temperatura média foram agrupados pelo grau de similaridade por meio da estatística multivariada utilizando a distância euclidiana e o método de ligação de Ward. Este foi utilizado devido à informação prévia do número de grupos desejáveis (período seco e úmido). A maioria das análises estatísticas foram realizadas no software Minitab versão 17.

Para avaliação dos eventos fenológicos foi utilizada a metodologia proposta por Fournier (1974), que se baseia na caracterização das fenofases (floração, frutificação, queda das folhas e brotação) e na intensidade das mesmas de acordo com as categorias semiquantitativas de cinco categorias, em que: $0=$ ausência do evento; 1 = ocorrência do evento entre 1 e $25 \% ; 2$ = ocorrência do evento entre 26 e $50 \% ; 3=$ ocorrência do evento entre 51 e $75 \%$ e 4 = ocorrência do evento entre 76 e $100 \%$.

Para análise de sincronismo dos eventos fenológicos foi medido o índice de atividade (ou índice de sincronia). Este método avalia a presença ou ausência da fenofase no indivíduo. Segundo Morellato et al. (1990), o índice de atividade tem caráter quantitativo em nível populacional, indicando a porcentagem de indivíduos da população que está manifestando determinado evento fenológico. Foram considerados assincrônicos (quando menos de $20 \%$ dos indivíduos se encontravam na fenofase); pouco sincrônicos ou sincronia baixa (quando de 20 a $60 \%$ dos indivíduos estavam na fenofase) e sincronia alta (quando mais de $60 \%$ dos indivíduos estavam na fenofase).

O valor que representa a amplitude da quinzena foi obtido por meio da metodologia proposta por Galetti, Pizo e Morellato (2006), na qual se faz a soma dos valores de amplitude obtidos para todos os indivíduos da espécie e divide-se pelo valor máximo possível (número de indivíduos multiplicado por quatro). Com isso obtém-se uma proporção, que é então multiplicada por 100.

Os dados quinzenais foram resumidos em valores médios mensais para cada fenofase, objetivouse mostrar a evolução de cada parâmetro e sua relação com os dados climáticos durante o período de observações (SANTOS; TAKAKI, 2005).

Para testar a normalidade dos dados foi realizado o teste de normalidade de Kolmogorov-Smirnov. Uma vez que os dados não apresentaram distribuição normal foi realizada a análise de correlação simples de Spearman $\left(r_{s}\right)$ para verificar a existência da ação dos fatores climáticos com os eventos fenológicos (GUEDES; QUIRINO; GONÇALVES, 2011), que foi escolhida pela natureza não paramétrica dos dados. Para isto, correlacionaram-se os dados de amplitude fenológica da espécie com os dados climáticos do mês de ocorrência e dos quatro meses anteriores, totalizando cinco períodos para correlação. Quanto mais próximo de 1 for o valor absoluto do coeficiente $\left(\mathrm{r}_{\mathrm{s}}\right)$, mais intensa é a relação linear entre as duas variáveis. 
O sinal negativo indica que as variáveis se correlacionam de maneira inversa, ou seja, o aumento de uma está correlacionado a diminuição da outra. A intensidade é classificada da seguinte maneira: se $\left|\mathrm{r}_{\mathrm{s}}\right|<0,20=$ a correlação é negligenciável; se $0,20<\left|\mathrm{r}_{\mathrm{s}}\right|<0,40=$ a correlação é fraca; se $0,40<\left|\mathrm{r}_{\mathrm{s}}\right|<0,60=\mathrm{a}$ correlação é moderada; se $0,60<\left|\mathrm{r}_{\mathrm{s}}\right|<0,80=\mathrm{a}$ correlação é forte; se $\left|\mathrm{r}_{\mathrm{s}}\right|>0,80=$ a correlação é muito forte.

Para detectar o comportamento sazonal da espécie, análises estatísticas circulares foram utilizadas, conforme descrito em Morellato, Alberti e Hudson (2010) e Bauer et al. (2012), utilizando o software Oriana versão 4.0, no qual os dias do ano foram convertidos em ângulos (dias do ano divididos por $360^{\circ}$ ) sendo 1 de janeiro o ângulo zero, em seguida esse valor angular diário foi associado à amplitude (\% Fournier) de cada evento fenológico para cada espécie.

Através da estatística circular foi possível testar o efeito das variações climáticas sobre a fenologia. Para tal, calculou-se a data ou ângulo médio $(\mu)$ de ocorrência e realizou-se o teste de Rayleigh $(Z)$ que determina o nível de significância do $\mu$. A hipótese nula é a de que os dados são distribuídos uniformemente ao longo do ano, e a hipótese alternativa é a de que os dados não são distribuídos aleatoriamente ao longo do ano. Caso a hipótese alternativa seja aceita, é calculado o vetor (r) que é utilizado como medida da magnitude do efeito da estacionalidade sobre a fenologia. Dessa forma, $r$ varia de 0 (a atividade fenológica é distribuída uniformemente ao longo do ano) a 1 (a atividade fenológica é concentrada em um período do ano) (NOGUEIRA et al., 2013).

\section{RESULTADOS E DISCUSSÃO}

Por meio da análise de agrupamento, considerando a linha de corte em -201,44 de similaridade, foi possível agrupar os dados meteorológicos nos grupos úmido e seco. Os dados revelaram que, para o período avaliado, a estação úmida ocupou um período maior que a estação seca (Figura 1), ocorrendo em geral de dezembro a fevereiro e de junho a agosto, respectivamente. Os demais meses foram classificados como períodos de transição aparecendo em ambos os grupos.

As maiores temperaturas e pluviosidades encontram-se no período úmido e os meses com elevados índices pluviométricos foram novembro e dezembro. A maior precipitação ocorreu em novembro de 2012 (258,4 mm) (Figura 2).

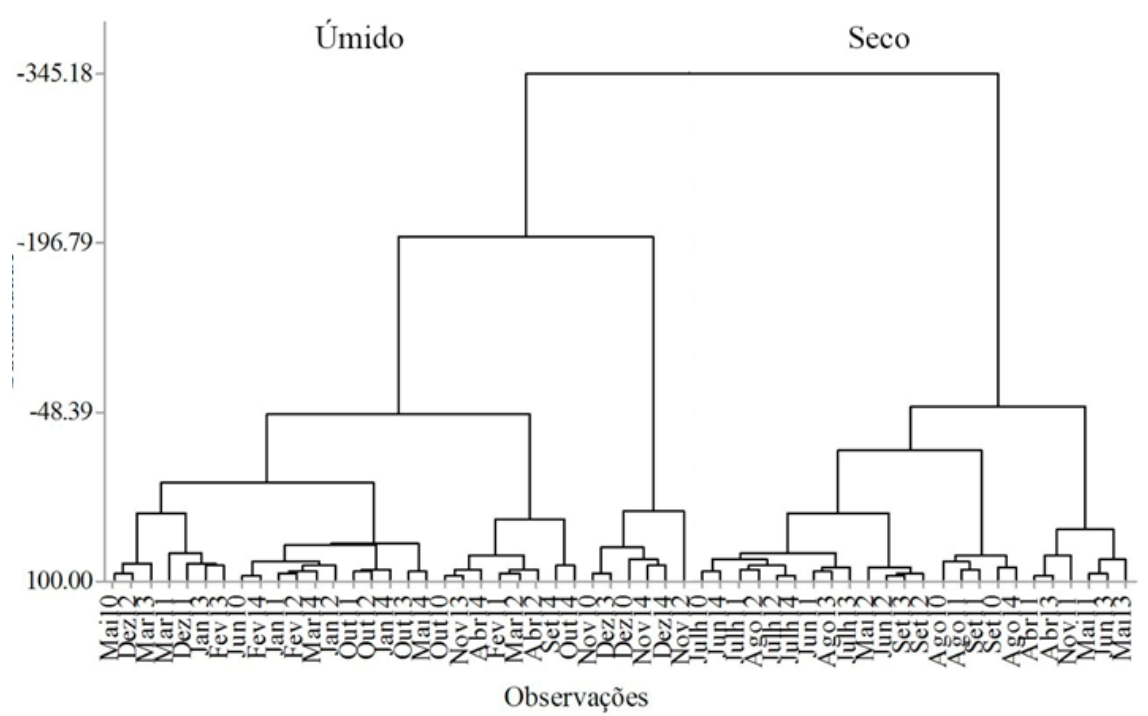

FIGURA 1: Dendrograma de agrupamento dos meses por meio do grau de similaridade entre as variáveis climáticas, utilizando os métodos de ligação de Ward e da distância euclidiana. As observações são demonstradas através da abreviação das três letras iniciais do mês mais a dezena do ano de ocorrência.

FIGURE 1: Grouping dendogram of the months by the similarity degree between climate variables, using the Ward connection method and Euclidian distance. The observations are demonstrated by the abbreviation of the first three letters of the month over the decade of the occurrence year. 

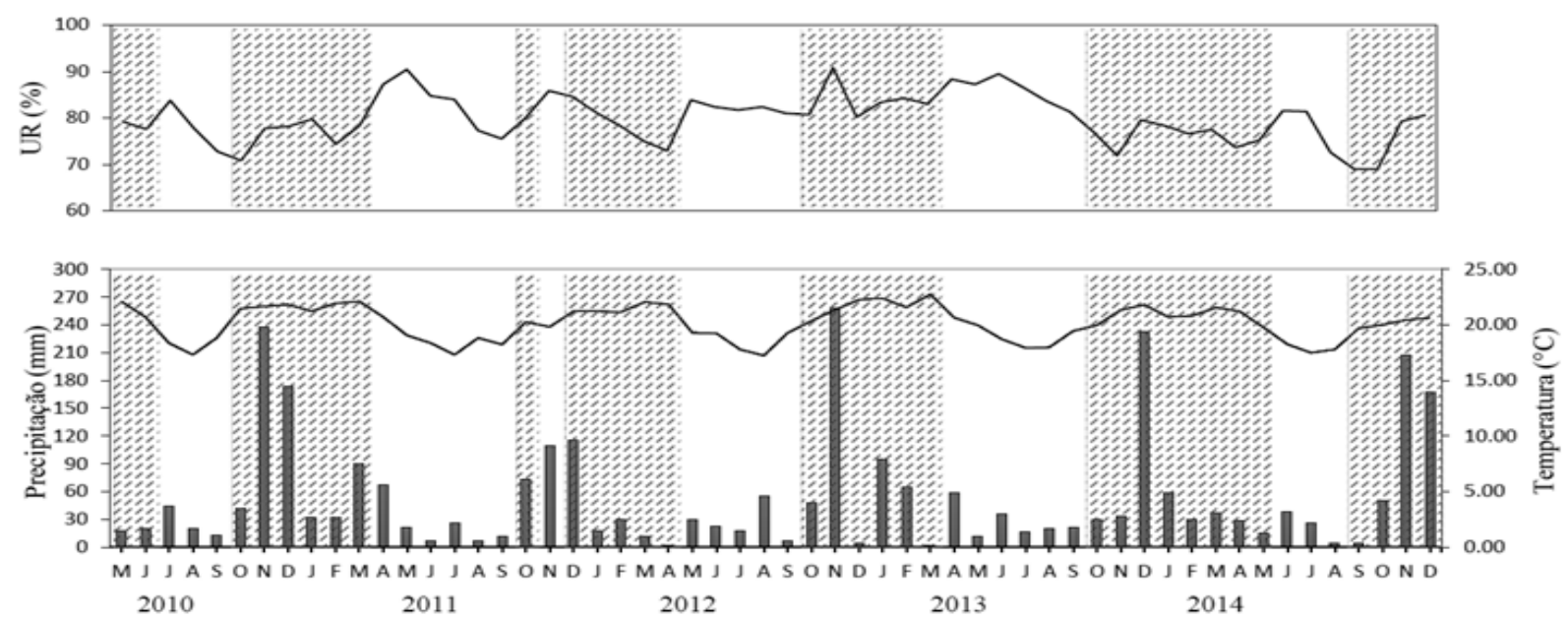

FIGURA 2: Precipitação total em mm (barras), temperatura média em ${ }^{\circ} \mathrm{C}$ (linha inferior) e umidade relativa em \% (linha superior) de maio de 2010 a dezembro de 2014 para município de Vitória da Conquista, Bahia, Brasil. O destaque tracejado indica os meses do período úmido obtido através da análise de agrupamento.

FIGURE 2: Total rainfall in mm (bar), average temperature in ${ }^{\circ} \mathrm{C}$ (bottom row) and Relative humidity in \% (top row) from May 2010 to December 2014 for the city of Vitória da Conquista, Bahia state, Brazil. The dashed highlight indicates the months of the wet period obtained through the cluster analysis.

A espécie apresentou queda foliar em diferentes intensidades durante todo o período, chegando a ficar completamente sem folhas nos períodos de pico (Figura 3), este padrão foi também encontrado por Morellato (1991) para Astronium graveolens, que descreveu como sendo do tipo decíduo. As maiores taxas de senescência ocorreram entre os meses de julho a outubro, podendo ir de maio a novembro considerando o desvio padrão (Tabela 1).

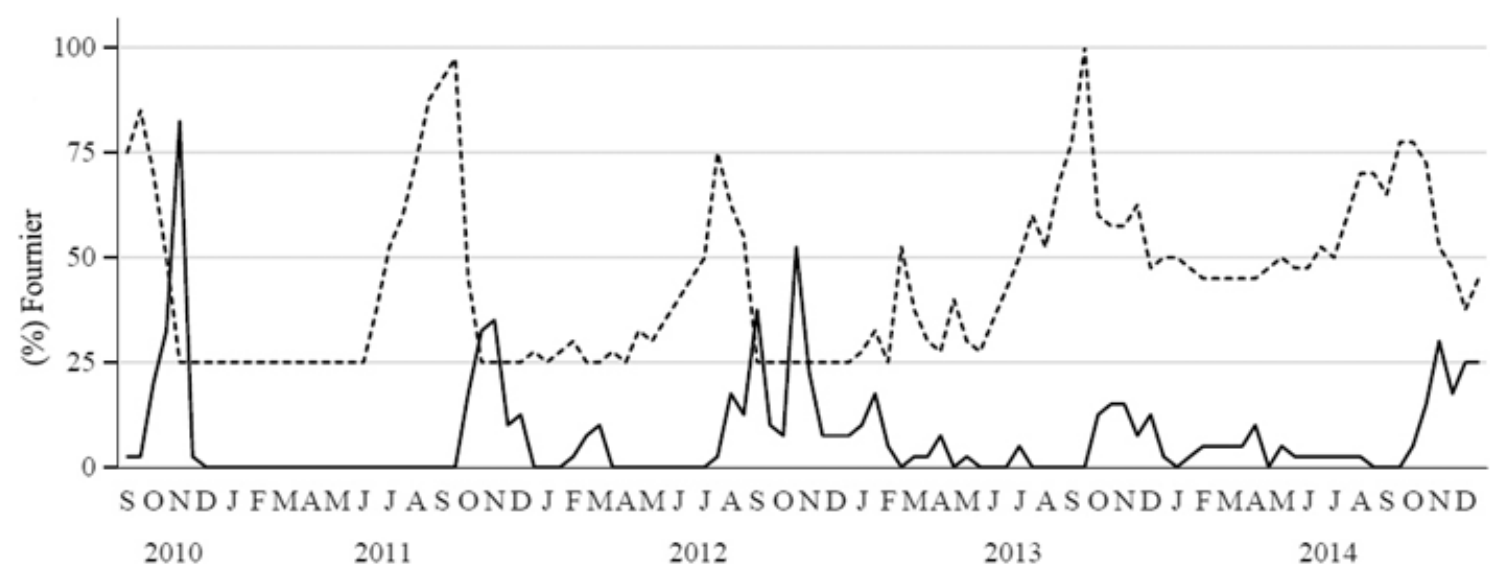

FIGURA 3: Fenograma da intensidade de Fournier para fenologia de Astronium graveolens Jacq.entre setembro de 2010 e dezembro de 2014, no município de Vitória da Conquista, Bahia, Brasil. A linha pontilhada representa a queda foliar e a linha continua o brotamento.

FIGURE 3: Phenogram of the Fournier intensity for phenology of Astronium graveolens Jacq. between September 2010 and December 2014, in the city of Vitória da Conquista, Bahia, Brazil. The dotted line represents the leaf fall and the continuous line represents the buds. 
Observou-se que, com exceção do ano de 2010, que apresentou menor número de observações devido à coleta ter sido realizada a partir de setembro (Tabela 1 ), o vetor (r) variou de 0,114 a 0,317 . Estes valores indicaram que mesmo havendo uma estacionalidade, esta apresentou-se em um intervalo longo, a exemplo de 2014 em que a senescência obteve um desvio padrão circular de $119,409^{\circ}$, que representa cerca de quatro meses. Resultado semelhante ao encontrado por Reich e Borchert (1984) e Paiva (2014), que relatam que a Astronium graveolens apresenta uma queda foliar que ocorre durante um longo período, raramente estando com a copa totalmente coberta de folhas.

TABELA 1: Resultados da análise de estatística circular para a ocorrência de sazonalidade nas fenofases de Astronium graveolens Jacq. em uma Floresta Estacional Decidual, Vitória da Conquista - BA.

TABLE 1: Results of the circular statistical analysis for the occurrence of seasonality in phenophases of Astronium graveolens Jacq. in deciduous forest, Vitória da Conquista, Bahia state.

\begin{tabular}{ccccccccc}
\hline Fase & Ano & Obs. & $\begin{array}{c}\text { Angulo } \\
\text { Médio }(\mu)\end{array}$ & $\begin{array}{c}\text { Desvio } \\
\text { Padrão } \\
\text { Circular }\end{array}$ & $\begin{array}{c}\text { Data } \\
\text { Média } \\
\text { Mès }\end{array}$ & $\begin{array}{c}\text { Desvio Padrão } \\
\text { Meses }\end{array}$ & $\begin{array}{c}\text { Vetor } \\
(\mathrm{r})\end{array}$ & $\begin{array}{c}\text { Teste de } \\
\text { Uniformidade } \\
\text { Rayleigh }(P)\end{array}$ \\
\hline QF & 2010 & 80 & $274,636^{\circ}$ & $31,887^{\circ}$ & Out & Set - Out & 0,857 & $<0,001$ \\
& 2011 & 240 & $225,963^{\circ}$ & $86,824^{\circ}$ & Ago & Jul - Out & 0,317 & $<0,001$ \\
& 2012 & 240 & $183,005^{\circ}$ & $103,03^{\circ}$ & $\begin{array}{c}\text { Jul } \\
\text { Mai - Ago }\end{array}$ & 0,199 & $<0,001$ \\
& 2013 & 240 & $255,536^{\circ}$ & $101,112^{\circ}$ & Set & Jul - Nov & 0,211 & $<0,001$ \\
BR & 2014 & 240 & $239,027^{\circ}$ & $119,409^{\circ}$ & Ago & Jul - Out & 0,114 & $<0,001$ \\
& 2010 & 21 & $290,972^{\circ}$ & $13,821^{\circ}$ & Out & Out & 0,971 & $<0,001$ \\
& 2011 & 21 & $294,648^{\circ}$ & $18,116^{\circ}$ & Out & Out - Nov & 0,951 & $<0,001$ \\
& 2012 & 37 & $273,419^{\circ}$ & $52,812^{\circ}$ & Out & Set - Out & 0,654 & $<0,001$ \\
& 2013 & 31 & $330,995^{\circ}$ & $62,068^{\circ}$ & Dez & Nov - Dez & 0,556 & $<0,001$ \\
& 2014 & 46 & $329,246^{\circ}$ & $63,462^{\circ}$ & Nov & Out - Dez & 0,541 & $<0,001$ \\
FL & 2010 & 3 & $272,364^{\circ}$ & $11,442^{\circ}$ & Out & Set - Out & 0,980 & $<0,001$ \\
& 2011 & 0 & - & - & - & - & - & - \\
& 2012 & 21 & $243,665^{\circ}$ & $30,180^{\circ}$ & Set & Ago - Set & 0,870 & $<0,001$ \\
& 2013 & 0 & - & - & - & - & - & - \\
& 2014 & 5 & $259,649^{\circ}$ & $14,395^{\circ}$ & Set & Set & 0,969 & $<0,001$ \\
FR & 2010 & 1 & $300,329^{\circ}$ & - & Nov & Nov & 1 & $<0,001$ \\
& 2011 & 0 & - & - & - & - & - & - \\
& 2012 & 8 & $317,594^{\circ}$ & $17,076^{\circ}$ & Nov & Nov & 0,957 & $<0,001$ \\
& 2013 & 2 & $000,493^{\circ}$ & - & Jan & Jan & 1 & $<0,001$ \\
& 2014 & 0 & - & - & - & - & - & - \\
\hline
\end{tabular}

Em que: Obs. = número de observações ao longo do ano; - = não aplicável devido à distribuição uniforme ou baixo número de observações; $\mathrm{QF}$ = queda foliar; $\mathrm{BR}$ = brotação; $\mathrm{FL}$ = floração; $\mathrm{FR}$ = frutificação.

A queda foliar correlacionou-se negativamente com a precipitação e a temperatura que ocorreram em até dois meses antes do evento, destacando-se a correlação negativa moderada com as chuvas do mês anterior $\left(r_{s}=-0,462\right)$ e a temperatura do mês de ocorrência $\left(r_{s}=-0,535\right)$. A umidade relativa teve fraca correlação negativa no mês de ocorrência $\left(\mathrm{r}_{\mathrm{s}}=-0,359\right)$ (Tabela 2). Este fato indicou que dois meses após as chuvas cessarem e no período em que se tem uma queda da temperatura, existe um aumento da abscisão foliar, comprovando que a espécie começa a perder suas folhas no início da estação seca. 
TABELA 2: Valores da análise de correlação de Spearman $\left(r_{s}\right)$ entre os fatores climáticos (precipitação total mensal, temperatura média mensal e umidade relativa média mensal).

TABLE 2: Values of Spearman correlation analysis $\left(r_{s}\right)$ between climatic factors (total monthly rainfall, average monthly temperature and relative humidity monthly average).

\begin{tabular}{lccccc}
\hline & & QF & BR & FL & FR \\
\hline Precipitação & 0 & $-0,277$ & 0,448 & $\mathrm{~ns}$ & $\mathrm{~ns}$ \\
& 1 & $-0,462$ & $\mathrm{~ns}$ & $\mathrm{~ns}$ & $\mathrm{~ns}$ \\
& 2 & $-0,284$ & $\mathrm{~ns}$ & $\mathrm{~ns}$ & $\mathrm{~ns}$ \\
Temperatura & 3 & $\mathrm{~ns}$ & $\mathrm{~ns}$ & $\mathrm{~ns}$ & $\mathrm{~ns}$ \\
& 4 & $\mathrm{~ns}$ & $\mathrm{~ns}$ & $\mathrm{~ns}$ & $\mathrm{~ns}$ \\
& 0 & $-0,535$ & $\mathrm{~ns}$ & $\mathrm{~ns}$ & 0,367 \\
Umidade Relativa & 1 & $-0,484$ & $\mathrm{~ns}$ & $-0,284$ & $\mathrm{~ns}$ \\
& 2 & $-0,304$ & $\mathrm{~ns}$ & $\mathrm{~ns}$ & $\mathrm{~ns}$ \\
& 3 & $\mathrm{~ns}$ & $-0,332$ & $\mathrm{~ns}$ & $\mathrm{~ns}$ \\
& 4 & $\mathrm{~ns}$ & $-0,412$ & $\mathrm{~ns}$ & $\mathrm{~ns}$ \\
& 0 & $-0,359$ & $\mathrm{~ns}$ & $\mathrm{~ns}$ & $\mathrm{~ns}$ \\
& 1 & $\mathrm{~ns}$ & $-0,274$ & $\mathrm{~ns}$ & $\mathrm{~ns}$ \\
& 2 & $\mathrm{~ns}$ & $\mathrm{~ns}$ & $\mathrm{~ns}$ & $\mathrm{~ns}$ \\
& 3 & $\mathrm{~ns}$ & $\mathrm{~ns}$ & $\mathrm{~ns}$ & $\mathrm{~ns}$ \\
& 4 & $\mathrm{~ns}$ & $\mathrm{~ns}$ & $\mathrm{~ns}$ & $\mathrm{~ns}$ \\
\hline
\end{tabular}

Em que: $0=$ mês de ocorrência do evento; $1,2,3$ e 4 = meses anteriores ao mês do evento fenológico de Astronium graveolens Jacq. $\mathrm{QF}=$ queda foliar; $\mathrm{BR}=$ brotação; $\mathrm{FL}=$ floração; $\mathrm{FR}=$ frutificação; $\mathrm{ns}=\mathrm{o}$ teste não apresentou correlação significativa para $\mathrm{P}<0,05$.

A queda foliar demonstrou ser um evento de alta sincronia (100\% em todo o período) (Tabela 3). Resultado semelhante ao de Paiva (2014) que encontrou uma sincrônica de 97\% para Astronium graveolens. Considerando que a amplitude chegou acima $80 \%$ nos meses de concentração, pôde-se confirmar que se trata de uma espécie de padrão decíduo adaptada para economia de água em períodos prolongados de seca. Resultado já esperado, pois é comum para espécies arbóreas decíduas a perda total de folhas durante a estação seca, estratégia adotada para evitar possíveis danos provocados pela escassez de água (LENZA; KLINK, 2006).

TABELA 3: Grau de sincronia por fenofase em percentagem de indivíduos estimado no período de máxima atividade das fenofases de Astronium graveolens Jacq., em Floresta Estacional Decidual, Vitória da Conquista BA.

TABLE 3: Degree of sync phenophase as a percentage of estimated individuals in the period of maximum activity of phenophases of Astronium graveolens Jacq. in Deciduous Forest, Vitória da Conquista, Bahia state.

\begin{tabular}{ccccc}
\hline Ano & QF & BR & FL & FR \\
\hline 2010 & 100 & 100 & 10 & 10 \\
2011 & 100 & 60 & 0 & 0 \\
2012 & 100 & 90 & 40 & 20 \\
2013 & 100 & 70 & 0 & 20 \\
2014 & 100 & 60 & 20 & 0 \\
\hline
\end{tabular}

Em que: $\mathrm{QF}=$ queda foliar; $\mathrm{BR}=$ brotação; $\mathrm{FL}=$ floração; $\mathrm{FR}=$ frutificação. 
O brotamento teve seu período de concentração de outubro a dezembro (Figura 3), podendo ir de setembro a dezembro considerando o desvio padrão (Tabela 1). Correlacionou-se de forma positiva moderada com a precipitação do mês em que ocorreu $(0,448)$; negativa moderada com a temperatura de quatro meses anteriores $(-0,412)$ e negativa fraca com a umidade relativa do mês anterior ao que ocorreu $(-0,274)$ (Tabela 2). Os dados indicaram que assim como em Reich e Borchert (1984) e Morellato (1991), a espécie começa a lançar folhas ao final da estação seca após as primeiras chuvas esporádicas que ocorrem na transição do período seco para o período chuvoso. Paiva (2014) relatou que o brotamento de Astronium graveolens apresentou alta correlação positiva com a temperatura do mês de ocorrência, porém, não encontrou correlação significativa com a precipitação para o mesmo período. Este fato pode indicar que em regiões de climas mais definidos com menor disponibilidade de chuvas a precipitação tenha maior influência no brotamento desta espécie. Diversos estudos realizados em florestas tropicais secas vêm demonstrando que a manifestação da incisão foliar acontece ao final da estação seca e início da estação úmida (SINGH; KUSHWAHA, 2005; ELLIOT; BAKER; BORCHERT, 2006; YADAV; YADAV, 2008).

A sincronia do brotamento variou de baixa em 2011 e 2014 (60\%) a alta nos anos de 2010 (100\%), 2012 (90\%) e 2013 (70\%) (Tabela 3). Demonstrou possuir de média a alta estacionalidade, como se pode ver através do comprimento do vetor (r) que variou de 0,541 a 0,971 (Tabela 1).

A espécie demonstrou ser de padrão supra-anual, especificamente bianual para a fenofase reprodutiva (Figura 4), com floração ocorrendo na data média mensal de outubro em 2010 e setembro em 2012 e 2014 (Tabela 1). Correlacionou-se de forma fraca e negativa com a temperatura média do mês anterior $(-0,284)$, e para as demais variáveis não houve correlação significativa (Tabela 2). Durante a coleta de dados em setembro de 2014 observou-se que o único indivíduo que havia produzido flores estava com alta intensidade de queda foliar e começando a formar os primeiros brotos, este fato corrobora as observações de Morellato (1991), que diz que a espécie produz suas flores e frutos quando esta se encontra ainda em alta intensidade de senescência. Foi um evento assincrônico em 2010 (10\%) e de baixa sincronia em $2012(40 \%)$ e 2014 (20\%) (Tabela 3). Altamente estacional com vetor (r) indo de 0,87 a 0,98 (Tabela 1 ) com poucos indivíduos manifestando a fenofase.

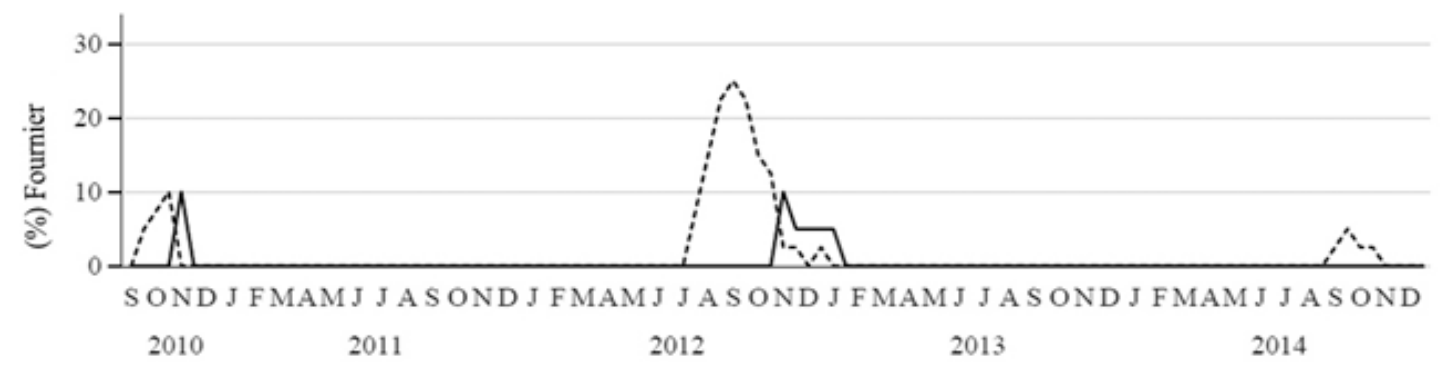

FIGURA 4: Fenograma da intensidade de Fournier para fenologia de Astronium graveolens Jacq., entre setembro de 2010 e dezembro de 2014, no município de Vitória da Conquista, Bahia, Brasil. A linha pontilhada representa a floração e a linha continua a frutificação.

FIGURE 4: Phenogram of the Fournier intensity for phenology of Astronium graveolens Jacq. between September 2010 and December 2014, in the city of Vitória da Conquista, Bahia state, Brazil. The dotted line represents the flowering and the continuous line the fruiting.

A frutificação aconteceu em novembro de 2010 e 2012 e janeiro de 2013, ocorrendo em um número ainda menor de observações quando comparada com a floração, teve correlação fraca e positiva com a temperatura do mês de ocorrência, não havendo correlação significativa com as demais variáveis climáticas (Tabela 1). O evento classificou-se como assincrônico em 2010 (10\%) e de baixa sincronia em 2012 e 2013 $(20 \%)$ e de alta estacionalidade (Tabelas 2 e 3 ). É importante destacar que os frutos observados em janeiro de 2013 são remanescentes dos frutos do ano anterior que estavam em fase de amadurecimento, sendo esta fase, portanto, de padrão bianual, o que difere do esperado e relatado por Morellato (1991) que a descreve como padrão anual. 
Diversos fatores bióticos influenciam diretamente a fase reprodutiva de espécies arbóreas, como, por exemplo, o tipo de reprodução e vetor de polinização. Por se tratar de uma espécie dióica e alógama, em que a polinização é em sua maioria entomófila realizadas por abelhas (ALLEM, 1991), a espécie Astronium graveolens necessita de condições específicas para poder gerar frutos em toda uma população, logo, a baixa oferta de flores aliada ao fato do sexo dos indivíduos deste estudo serem desconhecidos, contribuíram para a escassez de frutos. Cesario e Gaglianone (2008) relataram que a sincronia de florescimento entre plantas femininas e masculinas é fator imprescindível para o sucesso da polinização de espécies dióicas.

\section{CONCLUSÃO}

A espécie Astronium graveolens apresentou queda foliar de julho a outubro ocorrendo na estação seca, com alta sincronia e padrão decíduo. A brotação aconteceu de outubro a dezembro com sincronia alta na maior parte do estudo. A floração ocorreu de setembro e outubro, na transição do período seco para o úmido, com poucos indivíduos manifestando a fenofase e demonstrando baixa sincronia. A frutificação ocorreu de novembro a janeiro, no período úmido com baixa sincronia. A espécie demonstrou padrão reprodutivo bianual, sendo um resultado não esperado.

Não foram encontrados níveis fortes ou muito fortes de correlação entre as variáveis climáticas e a fenologia da espécie, de forma moderada tem-se que: a diminuição contínua da precipitação e o período de queda na temperatura estão correlacionados com o aumento da abscisão foliar, bem como o aumento da precipitação está correlacionado com o aumento do brotamento. A umidade relativa demonstrou ter fraca correlação no processo.

Este estudo fornece dados importantes para o planejamento de manejo da espécie e contribui com informações sobre a fenologia da espécie na região Nordeste. Trabalhos futuros com foco na fenologia reprodutiva da espécie, seus polinizadores e tipo dispersão de sementes devem ser realizados para complementar os resultados encontrados neste estudo.

\section{REFERÊNCIAS}

ALLEM, A. C. Estudo da biologia reprodutiva de duas espécies florestais (Aroeira e Gonçalo-alves) da região do cerrado. Embrapa-CENARGEN, Brasília, v. 2, p. 1-5, out. 1991.

BARRANCE, A. et al. Árboles de Centroamérica: un manual para extensionistas. 1. ed. Turrialba: Bib. Orton IICA; CATIE, 2003. 1079 p.

BAUER, D. et al. Fenologia de três espécies de Myrsine L. em floresta secundária semidecídua no Sul do Brasil. Revista Arvore, Viçosa, $M G$, v. 36, n. 5, p. 859-868, out. 2012.

BORCHERT, R. et al. Photoperiodic induction of synchronous flowering near the Equator. Nature, London, v. 433, p. 627-629, fev. 2005.

CESARIO, L. F.; GAGLIANONE, M. C. Biologia floral e fenologia reprodutiva de Schinus terebinthifolius Raddi (Anacardiaceae) em Restinga do Norte Fluminense. Acta Botanica Brasilica, São Paulo, v. 22, n. 3, p. 828-833, set. 2008.

ELLIOT, S.; BAKER, P. J.; BORCHERT, R. Leaf flushing during the dry season: the paradox of Asian monsoon forests. Global Ecologyand Biogeography, Malden, v. 15, n. 3, p. 248-257, maio 2006.

FOURNIER, L. A. Un método cuantitativo para la medición de características fenológicas en árbores. Turrialba, San José, v. 24, n. 4, p. 422-423, dez. 1974.

FOURNIER, L. A. Observaciones fenológicas en el bosque húmedo de premontano de Sanedro de Montes de Oca, Costa Rica. Turrialba, San José, v. 26, n. 1, p. 54-59. mar. 1976.

GAlETTI, M.; PIZO, M. A.; MORELlATO, P. C. Fenologia, frugivoria e dispersão de sementes. In:RUDRAN, R.; CULLEN JUNIOR, L.; VALLADARES-PADUA, C. Métodos de estudos em biologia da conservação e manejo da vida silvestre. 2. ed. Curitiba: Editora da UFPR; Fundação O Boticário de Proteção à Natureza, 2006. p. 395-422.

GUEDES, R. S.; QUIRINO, Z. G. M.; GONÇALVES, E. P. Fenologia reprodutiva e biologia da polinização de Canavalia brasiliensis Mart. Ex Benth (Fabaceae). Biotemas, Florianópolis, v. 22, n. 1, p. 27-37, 
ago. 2011

HERNANDEZ, V. et al. Chemical composition and antibacterial activity of Astronium graveolens Jacq. essential oil. Revista Latinoamericana de Química, Naucalpan de Juárez, v. 41, n. 2, ago. 2013. JESUS, R. B. Os recursos naturais e sua exploração na formação territorial do município de Vitória da Conquista - BA. Enciclopédia Biosfera, Goiânia, v. 6, n. 9, p. 1-13, 2010.

LENZA, E.; KLINK, C. A. Comportamento fenológico de espécies lenhosas em um cerrado sentido restrito de Brasília, DF. Revista Brasileira de Botânica, São Paulo, v. 29, n. 4, p. 627-638, dez. 2006.

LISI, C. S. et al. Tree-ring formation, radial increment periodicity, and phenology of tree species from a seasonal semi-deciduous forest in southeast Brazil. Iawa Journal, Leiden, v. 29, n. 2, p. 189-207, 2008.

LORENZI, H. Arvores brasileiras: manual de identificação e cultivo de plantas arbóreas nativas do Brasil. 5. ed. Nova Odessa: Plantarum, 2008. v. 1. 384 p.

LUCENA, I. C.; LEITE, M. B.; SILVA MATOS, D. M. A deciduidade foliar indica a vulnerabilidade de espécies lenhosas ao fogo. Revista Árvore, Viçosa,MG,v. 39, n. 1, p. 59-68, fev. 2015.

MORELLATO, L. P. C. As estações do ano na floresta. In: LEITÃO FILHO, H. F.; MORELLATO, L. P. C. Ecologia e preservação de uma floresta tropical urbana: Reserva de Santa Genebra. 1. ed. Campinas: UNICAMP, 1995. p. 187-192.

MORELLATO, L. P. C. Fenologia de árvores, arbustos e lianas em uma floresta semidecídua no sudeste do Brasil. 1991. Tese (Doutorado em Ecologia) - Universidade de Campinas, Campinas, 1991.

MORELlATO, L. P. C.; ALBERTI, L. F.; HUDSON, I. L. Applications of circular statistics in plant phenology: a case studies approach. In: HUDSON, I. L.; KEATLEY, M. Phenological research: methods for environmental and climate change analysis. 1. ed. Dordrecht: Springer, 2010. p. 357-371.

MORELLATO, L. P. C. et al. Estratégias fenológicas de espécies arbóreas em floresta de altitude na Serra do Japi. Revista Brasileira de Biologia, Jundiaí, v. 50, n. 1, p. 163-173, 1990.

NOGUEIRA, F. C. B. et al. Fenologia de Dalbergia cearenses Ducke (Fabaceae) em um fragmento de Floresta Estacional, no semiárido do Nordeste, Brasil. Revista Árvore, Viçosa, MG, v. 37, n. 4, p. 657-667, ago. 2013.

PAIVA, Y. K. Fenologia de espécies arbóreas em uma floresta estacional semidecidual no sul do Espírito Santo. 2014. 36 f. Monografia (Graduação em Engenharia Florestal) - Universidade Federal do Espírito Santo, Espirito Santo, 2014.

REICH, P. B.; BORCHERT, R. Water stress and tree phenology in tropical dry forest in the lowlands of Costa Rica. Journal of Ecology, London, v. 72, p. 61-74, mar. 1984.

SANTOS, D. L.; TAKAKI, M. Fenologia de Cedrela fissilis Vell. (Meliaceae) na região rural de Itirapina, SP, Brasil. Acta Botanica Brasilica, São Paulo, v. 19, n. 3, p. 625-632, set. 2005.

SILVA, C. B. M. C.; SANTOS, D. L. Fenologia reprodutiva de Melocactus conoideus Buin. \& Bred.: espécie endêmica do município de Vitória da Conquista, Bahia Brasil. Revista Brasileira de Biociências, Porto Alegre, v. 5, n. 2, p. 1095-1097, jul. 2007.

SINGH, K. P.; KUSHWAHA, C. P. Emerging paradigms of tree phenology in dry tropics. Current Science, Bengaluru, v. 89, n. 6, p. 964-975, set. 2005.

SOARES FILHO, A. O. Estudo Fitossociológico de Duas Florestas na Região Ecotonal no Planalto de Vitoria da Conquista, Bahia, Brasil. 2000. Dissertação (Mestrado em Ecologia) - Universidade de São Paulo, 2000.

YADAV, R. K.; YADAV, A. S. Phenology of selected woody species in a tropical drydeciduous forest in Rajasthan, India. Tropical Ecology, Varanasi, v. 49, n. 1, p. 25-34, 2008. 